\title{
Caractéristiques du sperme et potentiel d'utilisation par insémination artificielle des taureaux de race Borgou
}

\author{
M. Adamou N'Diaye ${ }^{1}$ R. Jondet ${ }^{2}$ A. Adjovi ${ }^{3}$
}

Mots-clés

Taurin Borgou - Insémination artificielle - Sperme - Saison humide Bénin.

\begin{abstract}
Résumé
Le but de ce travail est d'évaluer les caractéristiques principales du sperme de taureau Borgou en milieu tropical humide en vue de pratiquer l'insémination artificielle dans cette race. Les récoltes de sperme (au vagin artificiel) se sont déroulées pendant trois mois au cours de la grande saison des pluies au Bénin, au rythme d'un éjaculat par semaine pour 10 taureaux et de deux éjaculats par semaine pour 6 taureaux. Les résultats pour les éjaculats retenus lors de récoltes hebdomadaires sont en moyenne, volume $3,43 \mathrm{ml}$; motilité de masse 3,85 (échelle 0-5) ; motilité individuelle 75,7 p. 100 ; concentration $1,19 \times 10^{9}$ spermatozoïdes $/ \mathrm{ml}$; nombre total 4,09 × $10^{9}$ spermatozoïdes. lis sont, pour les éjaculats retenus lors de récoltes bihebdomadaires, volume 3,16 ; motilité de masse 4,30 ; motilité individuelle 79,5 p. 100 ; concentration $0,76 \times 10^{9}$ spermatozoïdes $/ \mathrm{ml}$; nombre total $2,45 \times 10^{9}$ spermatozoïdes.
\end{abstract}

\section{INTRODUCTION}

Le cheptel bovin de la République du Bénin s'élève à 1100000 têtes, dont 400000 appartiennent à la race Borgou*. Cette race, du nom du département du Borgou, résulte d'un croisement considéré comme stabilisé entre le taurin d'Afrique de l'Ouest à courtes cornes Somba (ou, plus accessoirement, Lagunaire) et le zébu, principalement le White Fulani (Mhororo Akou). I es hovins Borgou, que l'on rencontre aussi au Togo, au Burkina Faso et au Nigeria, sont des animaux trypanotolérants grâce à l'apport de sang Somba. Particulièrement dociles (qualité non négligeable), ils se révèlent compétitifs par rapport aux autres taurins d'Afrique de l'Ouest, eux aussi trypanotolérants (N'Dama, Baoulé), en matière de vitesse de croissance (23), de production laitière (18) et de taux de vêlage (10).

Des caractéristiques de sperme d'autres races voisines sont déjà connues (N'Dama, Baoulé), alors que celles de la race Borgou ne le sont guère (2). Pourtant, afin d'augmenter les productions de viande et de lait qui sont encore très insuffisantes, les autorités béninoises envisagent de promouvoir l'amélioration du bétail Borgou, notamment à l'aide de l'insémination artificielle. La connaissance des principales caractéristiques du sperme permet d'évaluer le potentiel d'utilisation d'un taureau au sein d'une population de vaches donnée au moyen de cette technique. Tel est l'objectif du présent travail.

1. Faculté des sciences agronomiques, UNB, BP 526, Cotonou, République du Bénin

2. Directeur général honoraire, Centre d'insémination artificielle, Rennes, France

3. Faculté des sciences agronomiques, UNB, BP 526, Cotonou, République du Bénin

* Chiffres fournis par la Direction de l'Elevage, Ministère du développement rural, Cotonou, République du Bénin

\section{MATERIEL ET METHODES}

Les 16 taureaux utilisés font partie de la station expérimentale d'élevage de la Faculté des sciences agronomiques (Université nationale du Bénin) à Cotonou. Ces taureaux, âgés de 4 à 6 ans, sont alimentés, à longueur d'année, avec les seuls pâturages locaux, c'est-à-dire les graminées qu'ils broutent pendant trois heures le matin et pendant deux heures et demie l'après-midi : Paspalum. conjugatum, Brachiaria defflexa, Sporobolus horizontalis, Eleusine indica, Panicum maximum, Andropogon gayanus. En dehors de ces périodes, ils sont maintenus au piquet, dans un enclos collectif pourvu d'un espace couvert. Ils n'y reçoivent aucune complémentation autre que des "pierres à lécher" (phosphate bicalcique, chlorures de sodium et de potassium, carbonate de zinc, sulfate de cuivre, soufre). Les taureaux de l'étude ont fait l'objet d'un sérieux entraînement préalable au prélèvement de sperme (sans difficulté) pendant plusieurs semaines, plusieurs mois pour certains. Les récoltes ont été effectuées le matin, entre 8 et 10 heures, à l'aide du vagin artificiel, le taureau concerné montant sur un "boutc-cn-train", un autrc mâlc cn l'occurrcnec, aprc̀s mise en conditionnement avec un temps de préparation de 10 min comprenant une ou deux fausses montes. Un seul éjaculat a été récolté par jour de collecte.

Le sperme recueilli a été aussitôt examiné à l'oeil nu, pour enregistrer le volume, la couleur et la consistance. Il a été ensuite observé au microscope, sur platine chauffante à $+37 /+38^{\circ} \mathrm{C}$ pour en apprécier la motilité. Pour la motilité massale, une goutte de sperme pur a été examinée au faible grossissement (40 à 100) : l'intensité et la turbulence des "vagues" ont été notêes selon l'échelle 0-5. Pour la motilité individuelle, une goutte de sperme dilué avec une solution tampon de citrate de sodium $2 \mathrm{H}_{2} \mathrm{O}(2,9 \mathrm{~g}$ dans $100 \mathrm{ml}$ d'eau bidistillée) a été observée entre lame et lamelle, au grossissement 300-400, pour évaluer la proportion de spermato- 
zoïdes vivants qui présentaient un mouvement de progression rectiligne vers l'avant (spermatozoïdes dits "fléchants"). La concentration a été déterminée avec précision à l'aide d'un hémocytomètre, par comptage des spermatozoïdes dans une goutte de sperme dilué au $1 / 100$ avec une solution de chlorure de sodium à 3 p. 100.

Ce travail, qui comporte deux parties, s'est déroulé sur une période de trois mois, en climat subéquatorial, pendant la grande saison des pluies (avril à juillet), alors que, dans cette zone côtière du Bénin, la température ambiante se maintient entre 28 et $31^{\circ} \mathrm{C}$ et que le taux d'humidité relative se situe entre 89 et $95 \mathrm{p}$. 100 .

Dans la première partie, dix taureaux ont été utilisés, à raison d'un seul prélèvement de sperme par semaine. N'ont été pris en considération que les éjaculats qui répondaient aux conditions requises pour une éventuelle utilisation en insémination artificielle, c'est-àdire ceux dont la motilité de masse était au moins égale à 3 (échelle 0-5), dont la motilité individuelle atteignait le minimum de 60 p. 100 de spermatozoïdes "fléchants", et dont la concentration n'était pas inférieure à $0,6 \times 10^{9}$ spermatozoïdes/ml.

Dans la deuxième partie, six autres taureaux ont été soumis à la fréquence de deux prélèvements de sperme par semaine au lieu d'un seul :

- le mardi et le jeudi (intervalle de $48 \mathrm{~h}$ ) pour trois d'entre eux,

- le mardi et le vendredi (intervalle de $72 \mathrm{~h}$ ) pour les trois autres.

\section{RESULTATS}

\section{Principales caractéristiques du sperme chez le taureau de race Borgou}

Avec un seul prélèvement de sperme par semaine

Les caractéristiques moyennes des 104 éjaculats retenus (sur 131 collectés, tous taureaux confondus) figurent au tableau I. On constate que la production "utile" par taureau présent s'est élevée à $4,09 \times 10^{9}$ spermatozoïdes par semaine.

\section{Avec deux prélèvements de sperme par semaine}

Lorsque les récoltes bihebdomadaires sont effectuées à 48 h d'intervalle (le mardi et le jeudi) ou lorsque l'intervalle qui sépare les deux récoltes est porté à $72 \mathrm{~h}$ (le mardi et le vendredi), les caractéristiques du sperme ne sont guère modifiées lors du deuxième prélèvement. Aucune différence ne s'est révélée significative $(\mathrm{p}<0,05)$. En totalisant les deux éjaculats, on a obtenu 5,39 x $10^{9}$ spermatozoïdes par taureau et par semaine avec un intervalle de 48 h et $4,42 \times 10^{9}$ spermatozoïdes par taureau et par semaine avec un intervalle de $72 \mathrm{~h}$.

\section{DISCUSSION}

\section{Principales caractéristiques du sperme chez le taureau de race Borgou}

Les seuils d'acceptabilité fixés par les auteurs en matière de motilité et de concentration ont entraîné, dans le cas des taureaux soumis à une récolte hebdomadaire, l'élimination de 27 éjaculats (20,6 p. 100). Cette élimination correspond à un minimum de sévérité dans un centre de production de semence, surtout lorsqu'il s'agit de semence congelée (14). Si ces mêmes seuils avaient été appliqués lors de récoltes bihebdomadaires, 14 éjaculats $(17,9$ p. 100) sur les 78 collectés auraient été ćliminćs : 4 pour motilité médiocre et 10 pour concentration insuffisante.

Les chiffres présentés ici concernant les caractéristiques du sperme chez le taureau Borgou sont un peu plus élevés que ceux rapportés pour le taureau Baoulé au Burkina Faso : volume 2,70 ml ; motilité de masse 3,10 ; concentration $0,96 \times 10^{9}$ spermatozoïdes $/ \mathrm{ml}$; nombre total de spermatozoïdes $2,59 \times 10^{9}$ (7). Les différences sont imputables à la race mais aussi, en partie tout au moins, à des facteurs saisonniers, du fait que ce travail de recherche n'a pu être

TABLEAu 1

Caractéristiques du sperme selon la fréquence des récoltes

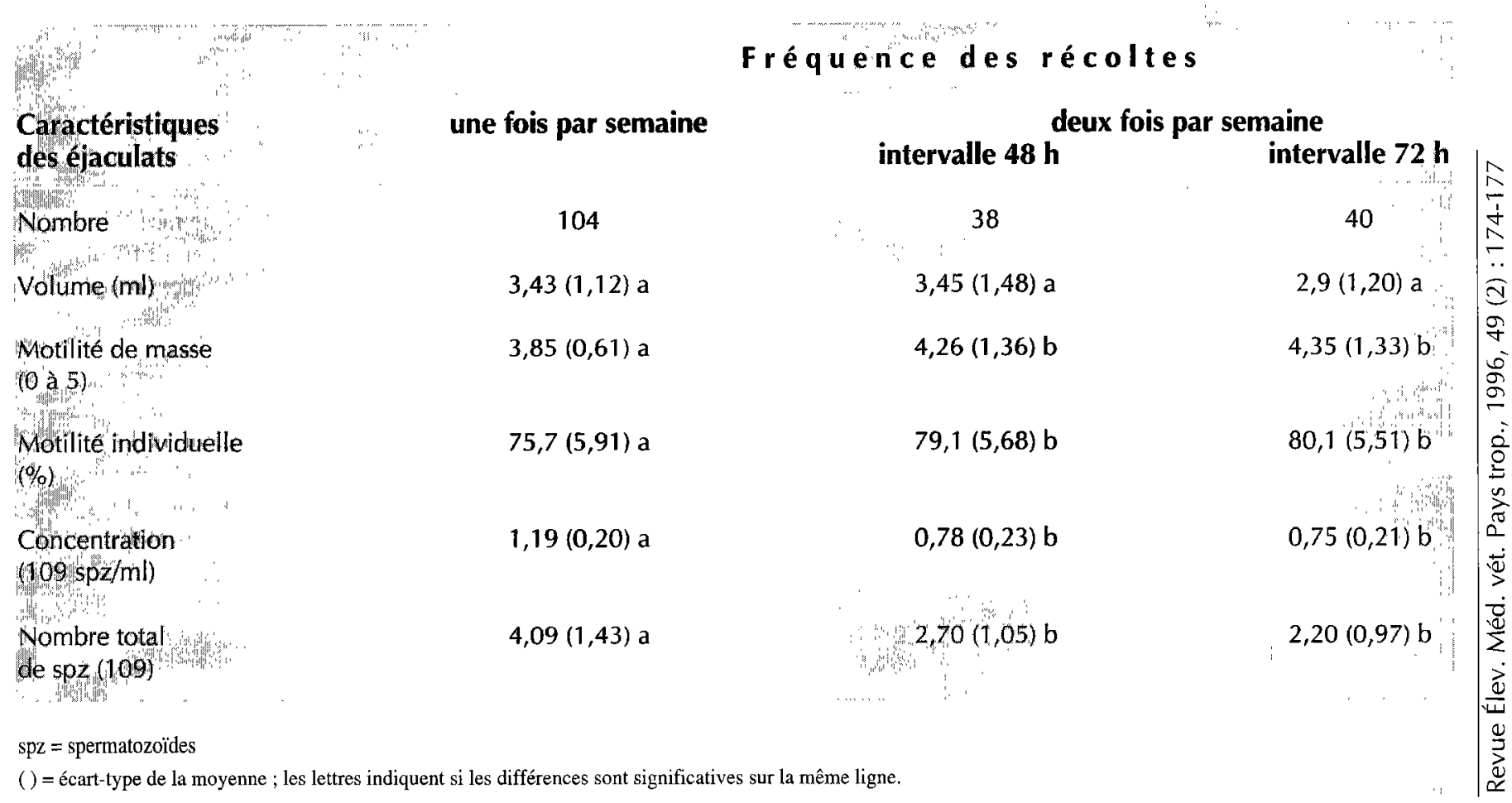


étalé sur toute une année. Les avis sont cependant partagés quant à l'influence du climat et de ses composantes (température ambiante, hygrométrie, insolation). La plupart des auteurs s'accordent plutôt sur le fait que les effets néfastes des fortes chaleurs se traduisent principalement par une augmentation des spermatozoïdes anormaux et ce, quelle que soit la race en cause $(11,12,16$, $20,22)$.

Le recours aux récoltes bihebdomadaires présente un intérêt certain en permettant d'obtenir davantage de spermatozoïdes d'un même taureau comparativement à une récolte hebdomadaire, dans le même temps. Ce gain avait été évaluć à 60 p. $100 \mathrm{avec}$ la racc Holstein (3).

\section{Potentiel d'utilisation d'un taureau Borgou pour l'insémination artificielle}

Sachant que la production de sperme (en quantité et en qualité) n'est pas égale toute l'année $(7,11,13,16,24)$, il a été supposé, pour les calculs, que la production annuelle correspond aux $4 / 5$ de celle obtenue en interpolant sur toute l'année les collectes effectuées en saison des pluies.

- Emploi de la semence diluée réfrigérée, conservée à $+5^{\circ} \mathrm{C}$ (semence dite "frâche")

Avec $10 \times 10^{6}$ spermatozoïdes par dose, la production hebdomadaire s'élève au moins à 400 doses de semence, soit environ 17000 par an. Des millions de vaches ont été fécondées avec de la semence "fraîche" au cours du demi-siècle écoulé, un peu partout dans le monde mais surtout en Europe et en Amérique du Nord. Des inséminations ont même été réalisées en Afrique (Kenya) sur des vaches zébu dès 1935 (4). Il faut dire que l'emploi de la semence "fraîche", dont le délai d'utilisation n'excède pas deux, trois jours tout au plus, est une technique simple qui ne requiert pas de matériel coûteux (21). Cette méthode qui, à titre expérimental, a déjà donné satisfaction au Bénin pourrait fort bien être adoptée dans ce pays, dans une première phase tout au moins.

- Emploi de la semence diluée congelée et conservée dans l'azote liquide $\left(-196^{\circ} \mathrm{C}\right)$

Les opérations de dilution (avec un milieu contenant du glycérol), de congélation et de dégel entraînent, par rapport à la semence "fraîche", une perte supplémentaire de spermatozoïdes. Pour obtenir une bonne fécondation, le nombre de spermatozoïdes mobiles après décongélation doit, comme pour la semence "fraîche", être compris entre 7 et 15 millions, ce qui correspond à un nombre total de spermatozoïdes initial pouvant aller de 10 à 60 millions de spermatozoïdes par dose de semence (8). En France, avec le sperme des taureaux de race laitière ou mixte, la race Normande, par exemple, chaque dose de semence conditionnée en minipaillettes contient le plus souvent 20 millions de spermatozoïdes et la proportion de spermatozoïdes trouvés "fléchants" au dégel atteint généralement des chiffres assez élevés : 65 p. 100 (moyenne de 12694 éjaculats), la perte restant au-dessous de 5 p. 100 (14). Il est vrai que, dans ce cas, les reproducteurs des centres d'insémination font l'objet d'une sélection poussée au point de vue zootechnique et qu'ils subissent en outre, lors de la puberté, un contrôle rigourcux de la fonction scxucllc (19). Ils sont, de plus, entretenus dans des conditions très strictes d'alimentation et d'hygiène. Il n'en est pas encore de même sous les tropiques où le taux de spermatozoïdes "fléchants" au dégel est, en général, de l'ordre de 40 à 45 p. 100 (ou parfois moins) et ce, quelle que soit la race du taureau : zébus (9), races d'origine européenne $(5,6)$, métis divers Bos indicus x Bos taurus $(15,17)$ et, naturellement, la race béninoise Borgou (1). Aussi, pour être sûr d'avoir dans la paillette, lors du dégel, le minimum souhaité de 15 millions de spermatozoïdes "fléchants", il importe de revoir à la baisse les chiffres de production de semence à partir d'un éjaculat. Tablant (prudemment) sur un taux de survie de 40 p. 100, il faudrait donc préparer des doses avec un total de $32,5 \times 10^{6}$ spermatozoïdes. On obtiendrait, en moyenne, 126 doses de semence congelée à partir de l'éjaculat récolté dans la semaine, soit environ 5200 par an.

- Combien de vaches un taureau Borgou pourrait-il féconder annuellement grâce à l'insémination artificielle ?

Dans les centres européens d'insémination artificielle, en France notamment, on considère qu'il ne faut pas moins de 1,6 à 1,7 inséminations, avec des techniciens parfaitement expérimentés, pour féconder une vache. Ce chiffre est souvent plus élevé en pays tropicaux où il peut même aller jusqu'à 3,6 et même plus.

Il semble donc raisonnable, dans un premier temps, de prévoir trois interventions afin d'éviter toute déconvenue. Un taureau Borgou pourrait alors féconder environ 5600 vaches par an avec de la semence "fraîche" et 1750 avec de la semence congelée.

La production des taureaux pourrait être cependant majorée :

1) en pratiquant deux prélèvements de sperme par semaine, au lieu d'un seul, sur un même taureau : le nombre de doses préparées par an passerait alors de 17000 à 22500 en semence "fraîche" et de 5200 à 7000 en semence congelée ; de ce fait, le nombre de vaches fécondées atteindrait 7500 avec de la semence "fraîche" et 2300 avec de la semence congelée.

2) en prélevant, sur un même taureau, deux éjaculats par jour de récolte ;

3) en améliorant l'entretien des reproducteurs, notamment en leur apportant une alimentation équilibrée de nature à favoriser la production du sperme, en quantité et en qualité ;

4) en les protégeant des excès de chaleur.

Des taureaux "de secours" sont, bien évidemment, indispensables surtout lors d'emploi exclusif de semence "fraîche" pour pallier les indisponibilités transitoires ou définitives.

De toutes façons, ces calculs n'ont d'autre prétention que celle de servir d'éléments d'estimation pour la création d'un service d'insémination artificielle.

\section{CONCLUSION}

Il découle de cette étude qu'il est possible de prélever du sperme, une ou deux fois par semaine, sur les taureaux de race Borgou, en saison des pluies. L'utilisation de l'insémination artificielle permettrait de mettre en ouvre un programme d'amélioration génétique.

\section{Remerciements}

Nous remercions bien sincèrement Monsieur le Professeur Labussière, de l'Ecole nationale supérieure agronomique de Rennes, et son collaborateur Monsieur Combaud, pour l'aide précieúse qu'ils nous ont apportée dans l'analyse statistique des résultats.

\section{BIBLIOGRAPHIE}

1. ADAMOU N'DIAYE M. 1994. Technologie du sperme chez le taureau de race Borgou. Thèse Doct. ès-Sciences, Université François Rabelais, Tours, France, 136 p. 
2. ADAMOU N'DIAYE M., JONDET R., ADJOVI A, DOSSOU BODJRENOU S.J., 1990. Caractéristiques du sperme chez le taureau de race Borgou. Bull. Acad. vét. France, 63 : 283-288.

3. ALMQUIST J.O., HALE E.B., 1957. An approach to the measurement of sexual behaviour and semen production of dairy bulls. In: 3 rd Cong. int. Reprod. Anim., Cambridge, United Kingdom, 25-30 juin 1956, p. 50-59.

4. ANDERSON J., 1945. The semen of animals and its use for artificial insemination. In: Proc. 7th int. Genet. Cong., 1939, Edimburgh. Edimbourg, United Kingdom, Tech. Commun. imp. Bureau Anim. Breed. Genet., p. 107-109.

5. BARNABE V.H., 1979. Avaliaçao de semen congelado de bovinos com especial referência à integridade do acrossomo. Thèse Doct., Faculdade de Medicina Veterinaria e Zootecnia, Universidade de Sao Paulo, Brésil, 70 p.

6. BONIA K.K., ANSARI M.R., BENJAMIN B.R., 1979. The effect of ambient temperature and relative humidity on the sperm recovery rate in frozen semen. Indian. vet. Med. J., 3: 59-64.

7. ClOE L.C., CHICOTEAU P., COULIBALY M., BASSINGA A., 1989. Caractéristiques spermatiques du taureau Baoulé Bos taurus taurus au Burkina Faso. Revue Elev. Méd. vét. Pays trop., 42 : 457-462.

8. CRAPLET C., THIBIER M., 1973. La vache laitière. Paris, France, Vigot Frères, 726 p. (p. 172).

9. DA SILVA R.G., CASAGRANDE J.F., 1977. Influence of high environmental temperatures on some characteristics of zebu semen, vol. IV. In: 8th Cong. int. reprod. anim. insem. artif., 12-16 juillet 1976, Cracovie, Pologne. Varsovie, Pologne, Ministère de l'agriculture, p. 939-942.

10. DEHOUX J.P., HOUNSOU-VE G., 1992. Productivité de la race bovine Borgou en milieu traditionnel au Nord-Est du Bénin. Rome, Italie, FAO. (Projet PNUD/FAO/BEN 886012 - Rapport d'étude n ${ }^{\circ} 1$ )

11. FAYEMI O., ADEGBITE O., 1982. Seasonal variations in sperm abnormalities in bulls in a tropical climate. Revue Elev. Méd. vét. Pays trop. 35 (1) : 69-72.

12. GAUTHIER D., VARO H., 1985. Caractéristiques spermatiques des taureaux en Guadeloupe. Variations avec la race et la saison. Ann. Zootech., 34 (4) : 463-470.

13. IGBOELI G., RAKHA A.M., 1971. Seasonal changes in the ejaculate characteristics of Angoni (short horn zebu) bulls. J. Anim. Sci, 33 (3): 651-654.

\section{Summary}

Adamou N'Diaye M., Jondet R., Adjovi A. Borgou bull semen characteristics and potential use in artificial insemination

A study was carried out in Benin subequatorial climate during the long rainy season to assess the potential for artificial insemination of Borgou bulls, taking into account semen characteristics. One ejaculate a week was collected from ten bulls and two a week from six bulls for three months. The results obtained for semen collected once a week were on average: volume $3.43 \mathrm{ml}$; mass motility 3.85 (0-5 scale); individual motility $75.7 \%$; sperm concentration $1.19 \times 10^{9}$ sperm $/ \mathrm{ml}$; total sperm $4.09 \times 10^{9}$. For semen collected twice a week, the results were: volume 3.16 ; mass motility 4.30 ; individual motility $79.5 \%$; concentration $0.76 \times 10^{9}$ sperm/ml; total sperm $2.45 \times 10^{9}$.

Key words: Borgou cattle - Artificial insemination - Semen Wet season - Benin.
14. JONDET R., 1980. Contribution à l'amélioration de la technologie du sperme de taureau. Thèse Doct. ès-Sciences, Université de Rennes, Rennes, France, $166 \mathrm{p}$.

15. JONDET R., PEREIRA ELER J., 1980. Tecnologia do sêmen. In : Reproduçao dos animais e inseminaçao artificial, $6 \mathrm{e}$ cd. Porto Alcgrc, Brésil, Livraria Sulina, $545 \mathrm{p}$.

16. KUMI-DIAKA J., OSORI D., NAGARATNAM U., 1980. Spermiograms of Sokoto Gudali bulls in relation to season and ration supplementation in Northern Nigeria. Br. vet. J., 136: 222-226.

17. MATHEWS A., JOSEPH P.J., JOSE T.K., 1982. Semen characteristics of purebred and cross-bred bulls. Indian vet. J., 59: 364-367.

18. OGODJA O.J., HOUNSOU-VE G., DEHOUX J.P., 1991. Impact de la supplémentation en graines de coton sur la qualité du lait trait et la croissance des veaux de vaches Borgou, et son intérêt économique. Parakou, République du Bénin, Centre d'action régionale de développement rural (CARDER). (Projet Développement pastoral intégré dans le Borgou)

19. PAREZ M., THIBIER M., 1983. Contrôle de la fonction sexuelle chez le jeune taurillon. Elev. Insém. $197: 3-16,198: 3-13$.

20. RAJA C.S.K., RAO A.R., 1983. Semen characteristics of Brown Swiss cross-bred bulls. Indian vet. J., 60: 23-28.

21. SAUVEROCHE B., 1993. La semence fraîche : une alternative à la semence congelée ? Relance de l'insémination artificielle en Afrique. In: I 'amélioration génétique des bovins en Afrique de I'Ouest'. Rome, Italie, FAO, p. 107-120. (Production et santé animales 110).

22. SEKONI V.O., GUSTAFFSON B.K., 1987. Seasonal variations in the incidence of sperm morphological abnormalities in dairy bulls regularly used for artificial insemination. Br. vet. J., 143: 312-317.

23. SINTONDJI B., 1986. De l'évolution pondérale du bovin africain sur pâturages naturels en milieu fermier. Cas des types Lagunaire et Borgou au ranch de Samiondji. Cotonou, Bénin, Direction de I'Elevage, Ministère du développement rural.

24. TAMBOURA H., TAHIRI-ZAGRET C., COULIBALY M., 1992. Influence du climat tropical humide sur les caractéristiques spermatiques des races taurines en Côte d'Ivoire. Bull. Anim. Health Prod. Afr., 40: $185-196$.

Reçu le 25.4.95, accepté le 11.9.96

\section{Resumen}

Adamou N'Diaye M., Jondet R., Adjovi A. Características del esperma y su potencial de utilización para la inseminación artificial, de los toros de raza Borgou

La finalidad del presente trabajo fue la de evaluar las características principales del esperma del toro Borgou en un medio tropical húmedo, con el fin de practicar la inseminación artificial en esta raza. Las colectas de esperma (gracias a una vagina artificial) se llevaron a cabo durante tres meses, en el curso de la gran estación lluviosa en Benin, con un ritmo de una eyaculación semanal en 10 toros y de dos eyaculaciones por semana para 6 toros. Los resultados de los eyaculados obtenidos durante las colectas semanales son, en promedio : volumen de $3,43 \mathrm{ml}$; motilidad en masa de 3,85 (escala de 0 a 5) ; motilidad individual de 75,7 p. 100 ; concentración de $1,19 \times 10^{9}$ espermatozoides $/ \mathrm{ml}$; cantidad total de espermatozoides de $4,09 \times 10^{9}$. Para los eyaculados obtenidos durante las colectas bisemanales son : volumen de 3,16 ; motilidad en masa de 4,30; motilidad individual de 79,5 p. 100 ; concentración de $0,76 \times 10^{9}$ espermatozoides $/ \mathrm{ml}$; cantidad total de espermatozoides de $2,45 \times 10^{9}$.

Palabras clave : Bovino Borgou- Inseminación artificial Semen - Estación húmeda - Benin. 